\title{
THE QUASIPOSITION REPRESENTATION IN THE SNYDER SPACE-TIME
}

\author{
B. Budnyy \\ Department for Theoretical Physics, \\ Ivan Franko National University of Lviv, \\ 12 Drahomanov St., Lviv UA-79005, Ukraine
}

(Received May 26, 2009)

\begin{abstract}
States with maximal spatial localization around the origin are found for the $(n+1)$-dimensional Snyder algebra. Maximally localized states in the Snyder space-time belong to the subset of the squeezed states in contrast to commutative algebra with minimal length. The noncommutativity of coordinate operators of the Snyder algebra leads to inhomogeneous space-time with the origin as the "center of the Universe".
\end{abstract}

Key words: Snyder algebra, squeesed states, minimal length.

PACS number(s): 03.65.Ca, 02.40.Gh, 11.30.Cp

\section{INTRODUCTION}

Modified canonical commutation relations have attracted much attention recently as a possible manifestation of more fundamental theories which are still under development (see, for example, [1-3]). In this paper the commutation relations with minimal length are considered. Their main feature is the nonzero minimal uncertainty of spatial coordinate operators. This property of the deformed commutation relations was the central motivation for Snyder's pioneer work [4], as he was looking for the regularization of UV divergences of quantum field theory. However, an attempt to develop electrodynamics over the Snyder space-time with minimal length had little success [5]. Field theory requires definition of fields and field equations while coordinates being not mere parameters but operators instead. The usual coordinate representation does not work here since the position eigenfunctions are unphysical. In [6] the so called quasiposition representation was proposed. When the fields (or quantum mechanical states) $\psi_{\mathrm{ml}}^{(x)}$ of maximal localization around any point $x$ of space are found one can define the quasiposition representation as follows

$$
\psi(x)=\left\langle\psi_{\mathrm{ml}}^{(x)} \mid \psi\right\rangle
$$

In [7] maximal localization states were studied for the Euclidean covariant algebra with minimal length. Another type of deformed commutation relations (the so called canonical noncommutativity, which breaks the Lorentz invariance) was considered in [8]. Some suggestions on the properties of the deformed Minkowski space-time were given in $[9,10]$. The two-parametric Lorentz covariant deformed algebra with minimal length and application to the Dirac oscillator was considered in [11]. In this paper the problem of quasiposition representation in the Snyder space-time is addressed.

\section{STATES OF MAXIMAL SPATIAL LOCALIZATION}

The definition of maximally localized states in multidimensional space is ambiguous. The obvious definition in the Euclidean case is the isotropic one [7], i. e., the state $\psi$ is maximally localized when the uncertainty $\sum_{i}\left\langle\psi\left|\left(\Delta X_{i}\right)^{2}\right| \psi\right\rangle$ is minimal. The Minkowski space is more involved to handle as the time coordinate is fundamentally different from spatial ones. Therefore "time-energy" commutation relation has negative sign in the deformation term and the corresponding uncertainty relation gives no minimal time [9]. Localization around some point (event) in space-time clearly breaks the Lorentz symmetry (except the degenerate case of infinite localization by the Dirac delta function in undeformed case). Here we consider the states which are maximally localized in space, thus minimizing the same functional as in the Euclidean case.

The $(n+1)$-dimensional Snyder algebra is given by the following commutation relations

$$
\begin{aligned}
& {\left[X^{\mu}, P^{\nu}\right]=-i \hbar\left(g^{\mu \nu}-\beta P^{\mu} P^{\nu}\right)} \\
& {\left[X^{\mu}, X^{\nu}\right]=-i \hbar \beta\left(P^{\mu} X^{\nu}-P^{\nu} X^{\mu}\right),} \\
& {\left[P^{\mu}, P^{\nu}\right]=0}
\end{aligned}
$$

where $g^{\mu \nu}=\operatorname{diag}(1,-1,-1, \ldots,-1), \beta$ is the deforming parameter, $X^{\mu}$ and $P^{\mu}$ are coordinate and momentum operators, respectively. Calculations will be performed in the momentum representation, the coordinate representation being unavailable due to nonzero minimal uncertainty in positions:

$$
X^{\mu}=-i \hbar\left(\frac{\partial}{\partial P_{\mu}}-\beta P^{\mu} P^{\nu} \frac{\partial}{\partial P^{\nu}}-\frac{3}{2} \beta P^{\mu}\right) .
$$

The operators thus defined are Hermitian with respect to the Lorentz covariant scalar product

$$
\langle\psi \mid \phi\rangle=\int d^{(n+1)} P \psi^{*}(P) \phi(P) .
$$

To start with, let us make the following (Lorentz noncovariant) transformation of the momentum variables:

$$
K^{\mu}=\frac{P^{\mu}}{\sqrt{1+\beta \mathbf{P}^{2}}}, \quad \mathbf{K}^{2} \in[0 ; 1 / \beta),
$$




\section{B. BUDNYY}

$\mathbf{P}$ being the spatial part of the momentum. Commutation relations of coordinate operators with $K^{\mu}$ operators read

$$
\left[X^{\mu}, K^{\nu}\right]=-i \hbar \sqrt{1-\beta \mathbf{K}^{2}}\left(g^{\mu \nu}-\beta g^{\mu 0} K^{0} K^{\nu}\right) .
$$

It is the feature of the Snyder algebra that such transformation leads to the separation of variables, that is in the $K$-representation the operators of the spatial coordinates $X^{i}$ have no dependence on $K^{0}$ :

$$
X^{i}=i \hbar\left(\sqrt{1-\beta \mathbf{K}^{2}} \frac{\partial}{\partial K^{i}}-\frac{\beta}{2} \frac{K^{i}}{\sqrt{1-\beta \mathbf{K}^{2}}}\right) .
$$

Let us find the symmetric states maximally localized in space around the origin with $\left\langle X^{i}\right\rangle=\left\langle P^{i}\right\rangle=0$. Following the procedure of [7] one can look for the squeezed state with minimal spatial uncertainty as a state of maximal localization. The corresponding equation is

$$
\left(X^{i}+i \alpha P^{i}\right) \psi=0,
$$

which in the $K$-representation reads

$$
\left[\frac{\partial}{\partial K^{i}}+\left(\frac{\alpha}{\hbar}-\frac{\beta}{2}\right) \frac{K^{i}}{1-\beta \mathbf{K}^{2}}\right] \psi=0 .
$$

Its general solution (containing arbitrary function of the $K^{0}$ variable) is

$$
\psi=f\left(K^{0}\right)\left(1-\beta \mathbf{K}^{2}\right)^{\alpha / 2 \hbar \beta-1 / 4} .
$$

The parameter $\alpha$ has to be found by minimization of $\left\langle(\Delta \mathbf{X})^{2}\right\rangle$. Rigorously speaking this method gives maximally localized solution from the subset of the squeezed states only, so one is not guaranteed to obtain the state localized as much as possible [12]. To prove that (10) is indeed an absolutely maximally localized state for some value of $\alpha$ we will directly minimize spatial uncertainty with additional condition for the states to belong to the domain on which an commutation relations (2) are represented. The minimization of $\left\langle(\Delta \mathbf{X})^{2}\right\rangle$ leads to the Euler-Lagrange equation (which is the eigenvalue equation of the $\mathbf{X}^{2}$ operator when $\left.\left\langle X^{i}\right\rangle=0\right)$ :

$$
\left(\mathbf{X}^{2}-\lambda^{2}\right) \psi=0
$$

The boundary condition is

$$
\left.\psi\right|_{\mathbf{K}^{2}=1 / \beta}=0,
$$

coming from the requirement for $\left\langle\left(P^{i}\right)^{2}\right\rangle$ to exist. In the $K$-representation equation (11) reads

$$
\left[\left(1-\beta \mathbf{K}^{2}\right)\left(\frac{\partial}{\partial \mathbf{K}}\right)^{2}-2 \beta \mathbf{K} \frac{\partial}{\partial \mathbf{K}}-\frac{\beta^{2} \mathbf{K}^{2}}{4\left(1-\beta \mathbf{K}^{2}\right)}-\frac{n \beta}{2}+\frac{\lambda^{2}}{\hbar^{2}}\right] \psi=0 .
$$

Due to the rotational symmetry the solution has the form $\psi=\psi\left(\mathbf{K}^{2}\right)$. After changing to dimensionless variable $q=\beta \mathbf{K}^{2}$ we have to solve the equation

$$
\left[4 q(1-q) \frac{d^{2}}{d q^{2}}+2(n-(n+2) q) \frac{d}{d q}-\frac{q}{4(1-q)}+\frac{\lambda^{2}}{\hbar^{2} \beta}-\frac{n}{2}\right] \psi=0,
$$

with

$$
q \in[0 ; 1],\left.\psi\right|_{q=1}=0
$$

Its general solution is expressed through the associated Legendre functions:

$$
\psi(q)=\frac{q^{1 / 2-n / 4}}{(1-q)^{1 / 4}}\left\{C_{1} P_{v}^{n / 2-1}(\sqrt{1-q})+C_{2} Q_{v}^{n / 2-1}(\sqrt{1-q})\right\},
$$

where the degree is

$$
v=\frac{1}{2}\left(\sqrt{(n-1)^{2}+\frac{4 \lambda^{2}}{\hbar^{2} \beta}}-1\right) .
$$

For our purpose, however, it is enough to look for the solution in the form $\psi(q) \sim(1-q)^{\delta}$. This particular solution and the corresponding eigenvalue of the $\mathbf{X}^{2}$ operator are

$$
\begin{aligned}
\psi & =f\left(K^{0}\right)\left(1-\beta \mathbf{K}^{2}\right)^{1 / 4}, \\
\lambda^{2} & =n \hbar^{2} \beta .
\end{aligned}
$$

It is easily checked that $\lambda$ coincides with the lower bound on the minimal length obtained from uncertainty relations for commutators (2). The commutation relations imply the minimal uncertainty of $\hbar \sqrt{\beta}$ for each spatial coordinate operator, therefore the lowest possible 
eigenvalue of the $\mathbf{X}^{2}$ operator is $n \hbar^{2} \beta$. So one concludes that (17) is the state of the maximal spatial localization for the Snyder algebra. One can further verify that (17) belongs to the set of squeezed states, namely, it is the squeezed state (10) with the parameter $\alpha=\hbar \beta$. A different situation arises in the case of commutative algebra with the minimal length of [7]. For this algebra the squeezed states are not the maximally localized ones (see [12]).

One may obtain the Lorentz covariant solution from the general expression (17) taking into account that

$$
1-\beta\left(K^{0}\right)^{2}=\frac{1-\beta P_{\nu} P^{\nu}}{1+\beta \mathbf{P}^{2}} .
$$

By choosing an arbitrary function $f\left(K^{0}\right)$ to be

$$
f\left(K^{0}\right)=\operatorname{const} \frac{1}{\left[1-\beta\left(\mathrm{K}^{0}\right)^{2}\right]^{\mathrm{n} / 4+1}},
$$

one gets the Lorentz covariant expression in the $P$ representation

$$
\psi(P)=\operatorname{const} \frac{1}{\left(1-\beta \mathrm{P}_{\nu} \mathrm{P}^{\nu}\right)^{\mathrm{n} / 4+1}} .
$$

State (20) is easily proved to be the squeezed state that saturates uncertainty relations for all the $n+1$ coordinate operators simultaneously. This solution is nonnormalizable and diverges on the hypersurface $P_{\nu} P^{\nu}=1 / \beta$. A similar divergence was observed in [11] for the generalized Lorentz covariant algebra with minimal length. The authors introduced the corresponding condition that restricts the energy of the physical states. The direct calculation of the bound states of $(1+1)$-dimensional Dirac oscillator showed that physical states exist for a finite range of deforming parameter only. The energy spectrum of the oscillator is bounded accordingly.

Normalizable states are allowed in (17) as well. For example, if one substitutes

$$
f\left(K^{0}\right)=\text { const } \frac{1}{\left[1+\beta\left(K^{0}\right)^{2}\right]^{\mathrm{n} / 4+1}},
$$

then in the $P$-representation

$$
\psi(P)=\operatorname{const} \frac{1}{\left(1+\beta\left(\mathrm{P}^{0}\right)^{2}+\beta \mathbf{P}^{2}\right)^{\mathrm{n} / 4+1}} .
$$

The states considered so far are localized around the origin. There is no simple algorithm how to obtain the states localized around an arbitrary point in the Snyder space-time. Since the coordinate operators do not commute the space-time is inhomogeneous. The usual translation operator does not exist in such a space-time. To show this one can start from the general Lorentz covariant form of the commutation relations

$$
\begin{aligned}
{\left[X^{\mu}, P^{\nu}\right] } & =i \hbar\left\{F\left(P^{2}\right) g^{\mu \nu}+G\left(P^{2}\right) P^{\mu} P^{\nu}\right\} \\
& =-i \hbar \Theta^{\mu \nu}(P) .
\end{aligned}
$$

The coordinate operators can be represented as

$$
X^{\mu}=-i \hbar \Theta^{\mu \sigma} \frac{\partial}{\partial P^{\sigma}}+f(P),
$$

$f(P)$ being an arbitrary function of momenta. The generator of space-time translations by a given values $\xi^{\mu}$, if it exists, is some function of momenta $T(P)$, which has to satisfy the following commutation relations

$$
\left[T, X^{\mu}\right]=\xi^{\mu}
$$

From this follows the equation for $T$ :

$$
\frac{\partial T}{\partial P^{\mu}}=\frac{i}{\hbar}\left(\Theta^{-1}\right)_{\mu \sigma} \xi^{\sigma}
$$

Since

$$
\frac{\partial^{2} T}{\partial P^{\mu} \partial P^{\nu}}=\frac{\partial^{2} T}{\partial P^{\nu} \partial P^{\mu}},
$$

one has to require

$$
\frac{\partial}{\partial P^{\nu}}\left(\Theta^{-1}\right)_{\mu \sigma}=\frac{\partial}{\partial P^{\mu}}\left(\Theta^{-1}\right)_{\nu \sigma}
$$

for the solution of (26) to exist. Being written in terms of the functions $F, G$ from (23), the last formula reduces to the Kempf condition [7] on coordinates commutativity:

$$
G=\frac{2 F F^{\prime}}{F-2 P^{2} F^{\prime}}
$$

One can of course still find the generators that would displace the mean values of coordinates in the state $\psi$. It is enough to require the following relation to satisfy, which is weaker than (25):

$$
\left\langle\psi\left|\left[T, X^{\mu}\right]\right| \psi\right\rangle=\xi^{\mu}
$$

However, the uncertainty of any coordinate in the transformed state $\psi^{\prime}=e^{-T} \psi$ will read

$$
\begin{aligned}
\left\langle\psi^{\prime}\left|\left(\Delta X^{\mu}\right)^{2}\right| \psi^{\prime}\right\rangle & =\left\langle\psi\left|\left(\Delta X^{\mu}\right)^{2}\right| \psi\right\rangle \\
& +\left\langle\psi\left|\left(\Delta\left[T, X^{\mu}\right]\right)^{2}\right| \psi\right\rangle .
\end{aligned}
$$

From this one concludes that the action of the displacement operator in the Snyder space-time results in the coordinate "smearing". This is equally true for any other noncommutative space with the Euclidean or Lorentz covariant commutators. There exists the "center of the Universe" in such a space which can be located with maximal precision. The space gets "smeared" while moving away from the center, that is, the minimal coordinate uncertainty grows with the distance to the origin. The displacement generator can be fitted to result in a sharp translation for any given direction, but the translation without smearing in one direction leads to the smearing in other directions. 


\title{
B. BUDNYY
}

\section{CONCLUSIONS}

In this paper the spatial localization properties of the Snyder space-time are considered. The states with minimal coordinate uncertainty centered around the origin are found. In contrast to deformed multidimensional algebra with minimal length and commuting coordinate operators the maximally localized states of the Snyder algebra belong to the set of squeezed states. There exists the Lorentz invariant solution which diverges on the hypersurface $P_{\nu} P^{\nu}=1 / \beta$. The similar divergence was earlier reported for a more general Lorentz covariant algebra with minimal length.

Due to the noncommutativity of the coordinate operators the Snyder space is inhomogeneous with the origin as the "center of the Universe". The coordinate uncertainty grows with the distance to the origin. The usual translation operator does not exist in such spaces so the definition and obtaining of the states localized in the vicinity of an arbitrary point needs further clarification. One could try to solve the variational problem of maximal localization with additional conditions that fix the mean values of coordinate operators. However, the separation of variables is unavailable for this problem. Alternatively one may apply the generalized deformed translations [11] to define the quasiposition representation.

\section{ACKNOWLEDGMENTS}

The author appreciates valuable discussions with V. M. Tkachuk, T. V. Fityo and Yu. S. Krynytskyi.
[1] L. J. Garay, Int. J. Mod. Phys. A 10, 145 (1995).

[2] E. Witten, Phys. Today 49, 24 (1996).

[3] R. J. Szabo, Class. Quant. Grav. 23, R199 (2006).

[4] H. S. Snyder, Phys. Rev. 71, 38 (1947).

[5] H. S. Snyder, Phys. Rev. 72, 68 (1947).

[6] A. Kempf, G. Mangano, R. Mann, Phys. Rev. D 52, 1108 (1995).

[7] A. Kempf, G. Mangano, Phys. Rev. D 55, 7909 (1997).
[8] M. Lubo, Phys. Rev. D 65, 066003 (2002).

[9] A. Kempf, Europhys. Lett. 40, 257 (1997).

[10] A. Kempf, Phys. Rev. Lett. 92, 221301 (2004).

[11] C. Quesne and V. M. Tkachuk, J. Phys. A 39, 10909 (2006).

[12] S. Detournay, C. Gabriel, Ph. Spindel, Phys. Rev. D 66, 125004 (2002).

\section{КВАЗІКООРДИНАТНЕ ПРЕДСТАВЛЕННЯ У ПРОСТОРІ-ЧАСІ СНАЙДЕРА}

\author{
Б. Будний \\ Кафедра теоретичної фізики, Львівсъкий націоналъний університет імені Івана Франка \\ e-mail: bohdan@ktf.franko.lviv.ua
}

\begin{abstract}
Для $(n+1)$-вимірної алґебри Снайдера знайдено стани, максимально локалізовані у просторі навколо початку координат. На відміну від комутативної алгебри з мінімальною довжиною, максимально локалізовані стани у просторі-часі Снайдера належать до множини стиснутих станів. Унаслідок некомутативності координатних операторів простір-час Снайдера є неоднорідним, у ньому існує “центр Всесвіту" в початку координат.
\end{abstract}

